\title{
Intracranial pressure monitoring in posterior fossa lesions-systematic review and meta-analysis
}

\author{
Sae-Yeon Won ${ }^{1}\left[\right.$ D Daniel Dubinski ${ }^{1} \cdot$ Jonas Hagemeier ${ }^{1} \cdot$ Bedjan Behmanesh $^{1} \cdot$ Svorad Trnovec $^{1}$. \\ Joshua D. Bernstock ${ }^{2} \cdot$ Thomas M. Freiman $^{1} \cdot$ Florian Gessler $^{1}$
}

Received: 7 December 2021 / Revised: 25 January 2022 / Accepted: 27 January 2022 / Published online: 3 February 2022

(c) The Author(s) 2022

\begin{abstract}
Elevated intracranial pressure (ICP) with reduced cerebral perfusion pressure is a well-known cause of secondary brain injury. Previously, there have been some reports describing different supra- and infratentorial ICP measurements depending on the location of the mass effect. Therefore, we aimed to perform a systematic review and meta-analysis to clarify the issue of optimal ICP monitoring in the infratentorial mass lesion. A literature search of electronic databases (PUBMED, EMBASE) was performed from January 1969 until February 2021 according to the Preferred Reporting Items for Systematic Reviews and Meta-analysis (PRISMA) statement. Two assessors are independently screened for eligible studies reporting the use of simultaneous ICP monitoring in the supra- and infratentorial compartments. For quality assessment of those studies, the New Castle Ottawa Scale was used. The primary outcome was to evaluate the value of supra- and infratentorial ICP measurement, and the secondary outcome was to determine the time threshold until equalization of both values. Current evidence surrounding infratentorial ICP measurement was found to be low to very low quality according to New Castle Ottawa Scale. Eight studies were included in the systematic review, four of them containing human subjects encompassing 27 patients with infratentorial pathology. The pooled data demonstrated significantly higher infratentorial ICP values than supratentorial ICP values $12 \mathrm{~h}$ after onset ( $p<0.05,95 \%$ CI 3.82-5.38) up to $24 \mathrm{~h}$ after onset ( $p<0.05$; CI 1.14-3.98). After $48-72 \mathrm{~h}$, both ICP measurements equilibrated showing no significant difference. Further, four studies containing 26 pigs and eight dogs showed a simultaneous increase of supra- and infratentorial ICP value according to the increase of supratentorial mass volume; however, there was a significant difference towards lower ICP in the infratentorial compartment compared to the supratentorial compartment. The transtentorial gradient leads to a significant discrepancy between supra- and infratentorial ICP monitoring. Therefore, infratentorial ICP monitoring is warranted in case of posterior fossa lesions for at least $48 \mathrm{~h}$.
\end{abstract}

Keywords Intracranial pressure monitoring · Posterior fossa lesion · Supratentorial ICP measurement · Infratentorial ICP measurement · Transtentorial gradient

Sae-Yeon Won

sae-yeon.won@med.uni-rostock.de

Daniel Dubinski

daniel.dubinski@med.uni-rostock.de

Jonas Hagemeier

jonas.hagemeier@uni-rostock.de

Bedjan Behmanesh

bedjan.behmanesh@med.uni-rostock.de

Svorad Trnovec

svorad.trnovec@med.uni-rostock.de

Joshua D. Bernstock

Jbernstock@gmail.com
Thomas M. Freiman

thomas.freiman@med.uni-rostock.de

Florian Gessler

florian.gessler@med.uni-rostock.de

1 Department of Neurosurgery, University Hospital Rostock, Schillingallee 35, 18057 Rostock, Germany

2 Department of Neurosurgery, Brigham and Women's Hospital, Harvard Medical School, Boston, USA 


\section{Introduction}

Increased intracranial pressure (ICP) with reduced cerebral perfusion pressure are long-established and well-known causes of secondary brain injury associated with poor clinical outcome [1]. Among a variety of ICP measuring devices, the most commonly used include ventricular catheter or intraparenchymal ICP probe. Irrespective of the location of the pathology or mass effect, it is a clinical practice that a single location for ICP measurement reflects an accurate pressure throughout the brain [2]. In view of the anatomic subdivision of our intracranial compartments by the falx or tentorium, the question arises if the ICP value is reliably independent from the location of the mass and ICP monitoring. Interestingly, the standardized clinical management of infratentorial mass lesions (cerebellar hemorrhage, stroke, tumor) relies on the supratentorial ventricular ICP measurement [3-5]. Previously, there have been several reports of patients, cadavers, and primates showing significant differences of ICP values depending on the location of mass and ICP monitoring. We therefore aimed to perform a systematic review and meta-analysis of the literature focusing on supraand infratentorial ICP measurements to clarify the issue of intracranial compartments and ICP monitoring in case of infratentorial mass lesions [6-14].

\section{Methods}

\section{Search strategy}

The meta-analysis was conducted in accordance with the Preferred Reporting items for Systematic Reviews and Metaanalysis (PRISMA) statement. We systematically searched the PubMed, Embase, Web of Science, and Cochrane databases including manuscripts published between January 1969 and February 2021, with language restricted to English, and identified all studies related to the use of simultaneously ICP monitoring in the supra- and infratentorial compartment. The literature was searched by using the predefined keywords "posterior fossa AND Intracerebral pressure monitoring," "infratentorial pressure monitoring," "posterior fossa pressure monitoring," "cerebellum AND intracranial pressure monitoring," and "cerebellar pressure monitoring."

\section{Data extraction and quality assessment}

Two assessors (S.W. and J.H.) independently screened for eligible studies by title and abstract. After prescreening, both assessors reviewed the full manuscripts of 27 eligible studies. Three studies in a language other than English, six studies without reporting of ICP values, and ten studies without simultaneous monitoring of supra- and infratentorial pressure monitoring were excluded. In total, eight studies were included in the systematic review, containing four studies with humans and four studies with primates (Fig. 1) [2, $7-12,14]$. Data were extracted in standardized data collection forms. The extracted information included the following items: first author name, year of publication, impact factor of the journal, sample size, species, pathology/illness, simultaneously supra- and infratentorial measured ICP values, and the duration of ICP monitoring. For the quality assessment of those studies, the New Castle Ottawa Scale was used by two independent reviewers.

\section{Outcome measures}

The primary outcome was to evaluate the value of supratentorial and infratentorial ICP measurement in different neurosurgical conditions. The secondary outcome was to determine the time threshold until equalization of both supra- and infratentorial ICP values. Therefore, we categorized the data into 3 time periods depending on the duration of ICP measurement $(0-12 \mathrm{~h}, 0-24 \mathrm{~h},>48-72 \mathrm{~h})$ and compared both ICP measurements.

\section{Statistical analysis}

Review Manager 5.4.1 (Cochrane Collaboration, Copenhagen, Denmark) was used to perform the meta-analysis. Supra- and infratentorial ICP values were summarized for the included studies using pooled weighted means with standard deviation (SD). The $\mathrm{I}^{2}$ statistic was used to reveal the heterogeneity of treatment effects. $\mathrm{I}^{2}$ of $0 \%$ indicates no heterogeneity, $25 \%$ indicates slight heterogeneity, $50 \%$ indicates middle heterogeneity, and $75 \%$ indicates high heterogeneity [15]. All statistical analysis were two-sided, and a $p$ value of $<0.05$ was considered as statistically significant.

\section{Results}

\section{Study characteristics}

Eight of 26 studies were included in the systematic review, four of them contained human subjects [7, 8, 11, 12]. Two studies were case series and the other two were case reports, which were included in the meta-analysis resulting in a total of 27 patients. All patients had an intracranial pathology in the infratentorial compartment as described in Table 1.

Four other studies contained animals in a preclinical experimental setting including a total of 26 pigs and eight dogs $[2,9,10,14]$. Of these four studies, three studies 
Fig. 1 Flowchart outlining of systematic review selection criteria

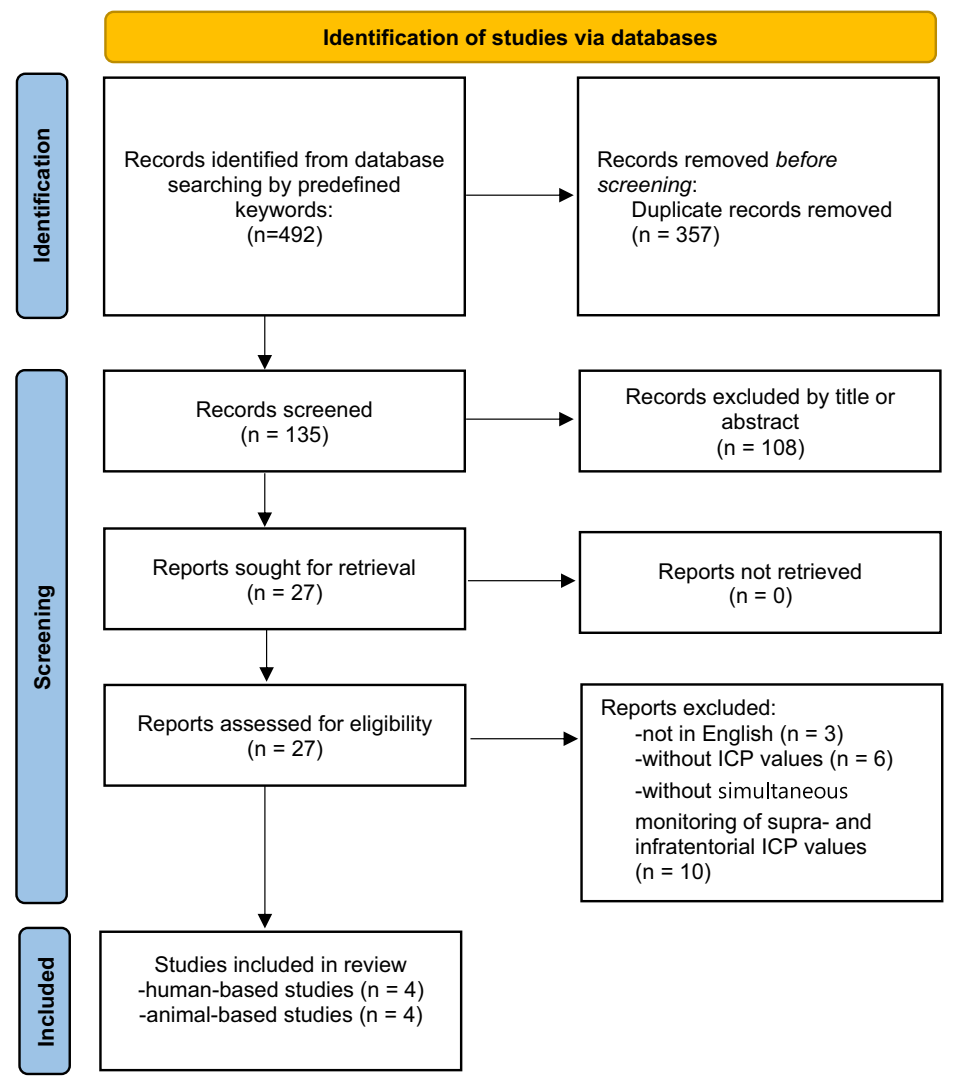

\section{Literature review}

The literature on Infratentorial ICP was searched on PUBMED by using keywords "Posterior fossa AND Intracerebral pressure monitoring", "Infratentorial pressure monitoring", "Posterior fossa pressure monitoring", "Cerebellum AND Intracranial pressure monitoring" and "Cerebellar pressure monitoring". Inclusion criteria was: simultaneously infra- and supratentorial ICP monitoring. Excluding criteria were: only infratentorial or supratentorial ICP monitoring and studies published not in English.

Table 1 Study characteristics (humans)

\begin{tabular}{|c|c|c|c|c|c|c|}
\hline References & Year & Design & Patients $(n)$ & Diagnosis & Quality (NOS) & $\begin{array}{l}\text { Impact factor } \\
\text { of Journal }\end{array}$ \\
\hline Rosenwasser et al. ${ }^{9}$ & 1989 & $\mathrm{CS}$ & 20 & $\begin{array}{l}\text { Vestibular schwannoma }(n=14) \\
\text { Meningioma in the cerebellopontine } \\
\text { angle }(n=4) \\
\text { Cerebellar hemangioblastoma } \\
(n=1) \\
\text { Cerebellar metastasis }(n=1)\end{array}$ & 5 & 4.1 \\
\hline Slavin et al. ${ }^{11}$ & 2003 & CS & 5 & $\begin{array}{l}\text { Cerebellar hemorrhage }(n=1) \\
\text { Subarachnoid hemorrhage of poste- } \\
\text { rior circulation }(n=2) \\
\text { Infratentorial arteriovenous malfor- } \\
\text { mation }(n=1) \\
\text { Infratentorial arachnoid cyst }(n=1)\end{array}$ & 4 & 2.4 \\
\hline Moyse et al. ${ }^{5}$ & 2016 & CR & 1 & Cerebellar infarct $(n=1)$ & N/A & 3.0 \\
\hline Khan et al. ${ }^{4}$ & 2020 & $\mathrm{CR}$ & 1 & Vestibular schwannoma $(n=1)$ & N/A & 1.3 \\
\hline
\end{tabular}

CS case series, $C R$ case report, NOS New Castle Ottawa Scale 
investigated mass lesions in the epidural frontal/temporal and basal ganglia region by the injection of autologous blood $[6,9,14]$. In one study, a balloon was installed in the cerebellum and was insufflated to imitate a mass lesion in the posterior fossa. Depending on the volume of the mass, the supratentorial (right frontal) ICP was compared with the infratentorial (cerebellar) ICP (Table 2) [10].

\section{Meta-analysis of supra- and infratentorial ICP measurement}

Three of four studies reported simultaneous ICP measurement up to $12 \mathrm{~h}$ after onset $(0-12 \mathrm{~h})$. Consequently, a total of 26 patients were included in this analysis [7, 11, 12]. The pooled data demonstrated that the mean value of infratentorial ICP measurement was significantly higher than the supratentorial ICP measurement $(\triangle \mathrm{ICP}=4.6 \mathrm{~mm}$ $\mathrm{Hg} 95 \%$ CI 3.82-5.38, $p<0.05, I^{2}=0 \%$ ) in favour for superiority of infratentorial measurement (Fig. 2a).

The second comparison with ICP measurement up to $24 \mathrm{~h}(0-24 \mathrm{~h})$ included all four human studies with a total number of 27 patients $[7,8,11,12]$. The pooled data demonstrated that there was again a significantly different mean value between supra- and infratentorial ICP $(\Delta \mathrm{ICP}=2.56 \mathrm{~mm} \mathrm{Hg} 95 \%$ CI $1.14-3.98, p<0.05$, $\left.I^{2}=66 \%\right)$ in favor for superiority of infratentorial measurement (Fig. 2b).

The third comparison with ICP measurement between 48 and $72 \mathrm{~h}$ included two studies with a total number of 21 patients $[7,11]$. The pooled data demonstrated a nonstatistically significant mean difference of $0.18 \mathrm{mmHg}$ ( $95 \% \mathrm{CI}-1.26$ to $0.9, p>0.05, I^{2}=0 \%$ ) between supraand infratentorial ICP measurement (Fig. 2c).

\section{Systematic review of supra- and infratentorial ICP measurement in an experimental setting}

The supratentorial ICP correlated well with the amount of the mass effect on epidural supratentorial compartment $[2$, $9,14]$. Increasing value of infratentorial ICP was shown as well; however, there was a significant mismatch between supra- and infratentorial ICP (range 0.9-90.4 mmHg) [2, 14]. Similar result could be shown in case of intracerebral mass effect with a significant difference between the mean value of supra- and infratentorial ICP $(42.1 \pm 3.5 \mathrm{mmHg}$ vs $29.1 \pm 4.5 \mathrm{mmHg} ; p=0.0009$ ) [9]. In contrast, one study showed no difference between supra- and infratentorial ICP measurement in case of mass lesion in the cerebellar compartment $(p>0.05)$ [10].

\section{Discussion}

The simultaneous supra- and infratentorial ICP measurement showed significant different values between those two compartments in human and in experimental models. The difference of those compartments persisted significantly in the first $48 \mathrm{~h}$ postoperatively, whereas after $48-72 \mathrm{~h}$, both values equilibrated without any significant difference.

This manuscript addresses a potentially relevant transtentorial gradient with clinical indication for additional intervention in the posterior fossa compartment. The outstanding question is to select right patient collective who might benefit from monitoring of posterior fossa compartment. According to the Monro-Kellie doctrine composed of brain, liquor, and blood, the intracranial space is limited and a mass lesion results in the reduction of liquor and blood component until the herniation of brain occurs [16, 17]. For the simplicity of this model, it is assumed that the pressure measured in a single location reflects the pressure

Table 2 Study characteristics (animals)

\begin{tabular}{|c|c|c|c|c|c|c|c|c|}
\hline References & Year & Type & (n) & Mass location & Lesion (cc) & $\begin{array}{l}\text { Supratentorial } \\
\text { ICP }(\mathrm{mmHg})\end{array}$ & $\begin{array}{l}\text { Infratentorial } \\
\text { ICP }(\mathrm{mmHg})\end{array}$ & $\begin{array}{l}\text { Impact factor } \\
\text { of Journal }\end{array}$ \\
\hline Wolfa et al. ${ }^{18}$ & 1996 & pig & 10 & Right epidural frontal & $\begin{array}{l}1 \\
2 \\
3 \\
4 \\
5 \\
6\end{array}$ & $\begin{array}{l}7.2 \\
10.3 \\
15.8 \\
24.8 \\
29.9 \\
41.6\end{array}$ & $\begin{array}{l}6.3 \\
7.4 \\
8.8 \\
9.9 \\
10.4 \\
12.3\end{array}$ & 4.0 \\
\hline Wolfa et al. ${ }^{17}$ & 1997 & Pig & 9 & Right epidural temporal & $\begin{array}{l}0 \\
3 \\
6\end{array}$ & $\begin{array}{l}10.5 \\
59,8 \\
147.9\end{array}$ & $\begin{array}{l}9.7 \\
39.2 \\
57.5\end{array}$ & 4.0 \\
\hline Rieger et al. ${ }^{8}$ & 1999 & Pig & 7 & Left cerebellar & $\begin{array}{l}0 \\
\text { Balloon infl }\end{array}$ & $\begin{array}{l}4.1 \\
63.1\end{array}$ & $\begin{array}{l}4.4 \\
62.3\end{array}$ & 1.3 \\
\hline Qureshi et al. ${ }^{7}$ & 2002 & Dog & 8 & Right basal ganglia & 5 & $42.1 \pm 3.5$ & $29.1 \pm 4.5$ & 7.6 \\
\hline
\end{tabular}

ICP intracranial pressure 
A. $0-12$ hours after onset

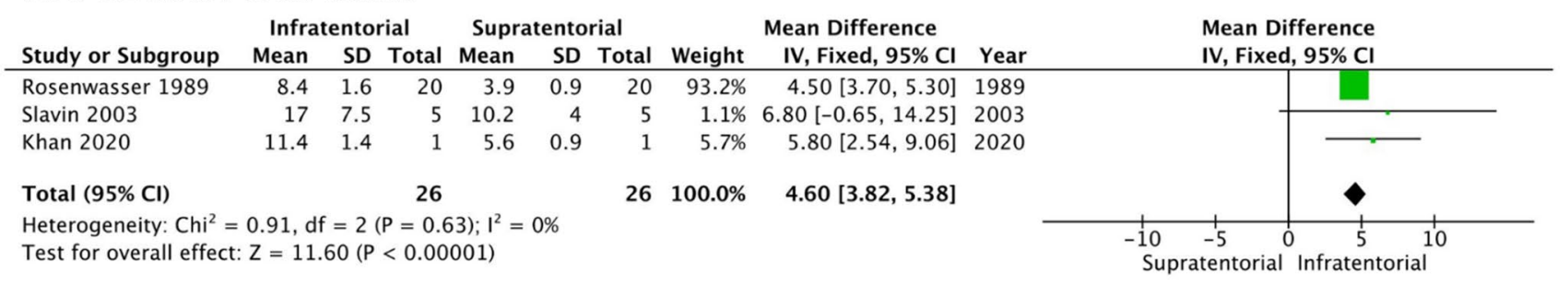

B. 0-24 hours after onset

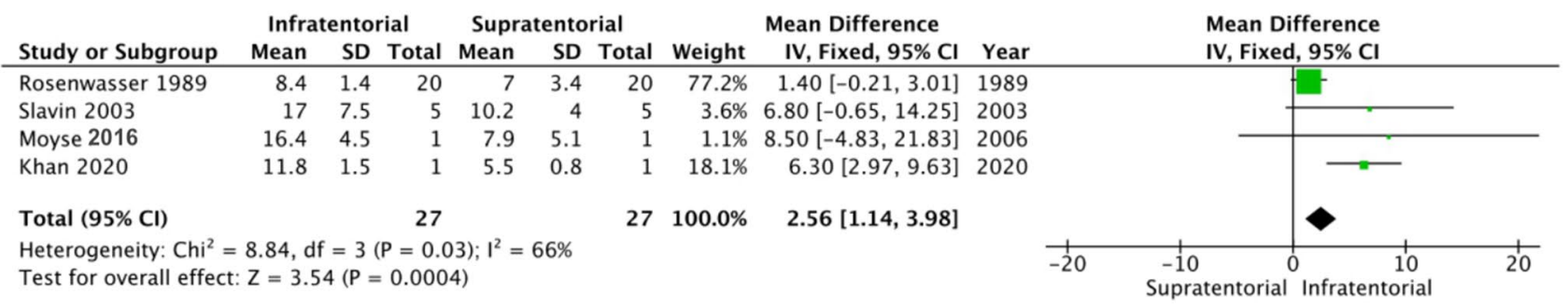

\section{48-72 hours after onset}

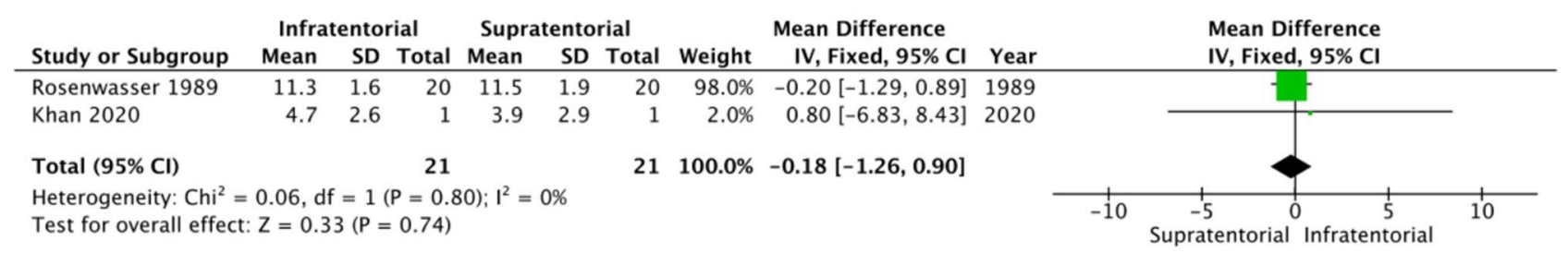

Fig. 2 Meta-analysis comparing supra- and infratentorial intracranial pressure monitoring

throughout the brain. However, several manuscript as well as our review show that there is a significant difference between supra- and infratentorial ICP in humans depending on the location of the lesion, volume of the lesion, and time after onset of the lesion $[7,8,11,12]$. Similar results were shown in experimental studies including animals except for one study reporting non-significance of supra- and infratentorial ICP measurements $[2,9,10,14]$. Not only between supraand infratentorial compartment but also the more remotely ICH was measured, the more significant ICP difference occurred. For example, in case of mass lesion in the frontal lobe in an experimental setting, the ICP value decreased as follows: frontal lobe $>$ temporal lobe $>$ midbrain $>$ cerebellum [2]. Accordingly, the meaning of infratentorial pressure monitoring appears to be both important and relevant in the clinical management of posterior fossa lesions, the larger the mass lesion the more important the investigation of posterior fossa ICP monitoring. One should be alert that a normal supratentorial ICP could mistakenly mask the pressurerelated secondary injury in the posterior fossa region. Still, a supratentorial ICP monitoring is necessary due to its risk of development of hydrocephalus by obstruction of fourth ventricle. At the end, the composition of those pathophysiological mechanisms is the determining factor for the final outcome of patients.

Further, the type of mass lesion and the preoperative neurological status might be crucial for the indication of ICP monitoring in the posterior fossa region. Cerebellar hemorrhage or infarction is lesion with increased risk for postoperative edema development or rebleeding compared to elective tumor surgery, which might be appropriate for an infratentorial ICP monitoring. In addition, good preoperative neurological status is a reliable parameter to compare the postoperative result independent from an ICP monitoring, whereas worse preoperative neurological status might end up in difficulty to evaluate the actual status of patients. We think that those patients might benefit from an ICP monitoring in the posterior fossa region.

Even focusing on the supratentorial compartments alone, there are contrary reports in the literature concerning interhemispheric gradient. Several studies observed significantly higher ICP values on the ipsilateral side of the mass lesions compared to the contralateral side concluding the existence of interhemispheric ICP gradients. With time, the gradient 
disappeared and both values equilibrated [2, 18, 19]. In contrast, Yano et al. observed bifrontal ICPs in patients with traumatic brain injury showing no significant difference in the concurrent comparative ICPs concluding that the supratentorial space be assumed as one compartment regardless of different types of intracranial lesions [20]. As a limitation of this study, the observed range of ICPs was between -3 and $130 \mathrm{mmHg}$. Based on the results, it again strengthens the hypothesis that two factors are relevant concerning the gradient between different compartments: the size of mass lesion and time after onset of the lesion.

After a period of time, the intracranial pressure in different compartment begins to equilibrate, and according to our meta-analysis, the threshold was between 24 and $48 \mathrm{~h}$ after onset. In other words, we have a blind window of $48 \mathrm{~h}$ without showing the actual pressure of posterior fossa compartment if only a supratentorial ICP measurement is performed. The aspects unique to the posterior fossa include the limited volume, a close relationship to the brain stem, and a high probability of development of occlusive hydrocephalus. Giving the truth of insufficient supratentorial ICP measurement for $48 \mathrm{~h}$, secondary brain injury in this region could lead to a fatal outcome. Therefore, a simultaneous monitoring of supra- and infratentorial ICP measurement is important at least for $48 \mathrm{~h}$ or more in patients with mass lesion in the posterior fossa. The time window might depend on the underlying pathology (cerebellar tumor, hemorrhage, infarction) and volume of the mass lesion, wherefore further studies need to be investigated to evaluate the necessary time window for simultaneous ICP measurement.

As reported, there are several studies reporting a difference in pressures between various compartments of the brain. However, there are no studies reporting the relationship between infratentorial ICP monitoring and the outcome of patients. This should be addressed in future studies to establish an evidence-based treatment strategy for posterior fossa pathologies to promote better ICP monitoring and outcome of our patients.

\section{Limitations}

This study contains human and animal studies, which are mostly based on case series or case reports. Accordingly, the quality of included studies is limited. However, the main conclusions of those studies are uniform which show indirectly the relevance of further prospective studies to evaluate this issue more in detail. Secondly, the simultaneous ICP measurement was performed in different pathologies in the posterior fossa region. This is an important point, since the difference of supra- and infratentorial ICP measurement as well as the time window until the pressure equilibration might be more prominent if the pathology of posterior fossa region was more space-occupying.

\section{Conclusions}

Supratentorial ICP measurement is not a reliable tool to reflect the pressure in the posterior fossa. Therefore an infratentorial ICP monitoring might be mandatory to reflect the real pressure in the posterior fossa region to prevent further secondary brain injury. Simultaneous supra- and infratentorial ICP measurement should be performed at least for $48 \mathrm{~h}$ or more until the pressure begins to equilibrate.

Author contributions S.W. and J.H. conceived and designed the analysis, collected the data, performed the analysis, and wrote the paper. D.D., B.B., J.B., and T.F. were involved in drafting the manuscript and revising it critically for important intellectual content. F.G. designed the analysis, performed analysis and interpretation of data, and supervised this study.

Funding Open Access funding enabled and organized by Projekt DEAL.

Data availability The datasets used and/or analyzed during the current study are available from the corresponding author on reasonable request.

Code availability Not applicable.

\section{Declarations}

Ethics approval This study was approved by the local ethic committee of University Hospital Rostock (Registration-Nr. A 2020-0276).

Consent to participate Informed consent of patient was waived for this systematic review and meta-analysis.

Consent for publication Not applicable.

Conflict of interest J.B. has positions and equity in CITC Ltd and Avidea Technologies and is on the Scientific Board of Advisors for POCKiT Diagnostics.

Open Access This article is licensed under a Creative Commons Attribution 4.0 International License, which permits use, sharing, adaptation, distribution and reproduction in any medium or format, as long as you give appropriate credit to the original author(s) and the source, provide a link to the Creative Commons licence, and indicate if changes were made. The images or other third party material in this article are included in the article's Creative Commons licence, unless indicated otherwise in a credit line to the material. If material is not included in the article's Creative Commons licence and your intended use is not permitted by statutory regulation or exceeds the permitted use, you will need to obtain permission directly from the copyright holder. To view a copy of this licence, visit http://creativecommons.org/licenses/by/4.0/. 


\section{References}

1. Smith M, Maas AIR (2019) An algorithm for patients with intracranial pressure monitoring: filling the gap between evidence and practice. Intensive Care Med 45:1819-1821. https://doi.org/10. 1007/s00134-019-05818-4

2. Wolfla CE, Luerssen TG, Bowman RM, Putty TK (1996) Brain tissue pressure gradients created by expanding frontal epidural mass lesion. J Neurosurg 84:642-647. https://doi.org/10.3171/jns. 1996.84.4.0642

3. Gerber LM, Chiu Y-L, Carney N, Härtl R, Ghajar J (2013) Marked reduction in mortality in patients with severe traumatic brain injury. J Neurosurg 119:1583-1590. https://doi.org/10.3171/ 2013.8.JNS13276

4. Neugebauer H, Witsch J, Zweckberger K, Jüttler E (2013) Spaceoccupying cerebellar infarction: complications, treatment, and outcome. Neurosurg Focus 34:E8. https://doi.org/10.3171/2013.2. FOCUS12363

5. Tewari MK, Tripathi M, Sharma RR, Mishra GP, Lad SD (2015) Surgical Management of Moderate Sized Spontaneous Cerebellar Hematomas: Role of Intracranial Pressure Monitoring. Turk Neurosurg 25:712-720. https://doi.org/10.5137/1019-5149.JTN. 11011-14.1

6. Czosnyka M (2004) Monitoring and interpretation of intracranial pressure. J Neurol Neurosurg Psychiatry 75:813-821. https://doi. org/10.1136/jnnp.2003.033126

7. Khan A, Borg N, Shenouda E (2021) Posterior fossa ICP monitoring: a tale of two compartments. Br J Neurosurg 35:129-132. https://doi.org/10.1080/02688697.2020.1765974

8. Moyse E, Ros M, Marhar F, Swider P, Schmidt EA (2016) Characterisation of supra- and infratentorial ICP profiles. pp 37-40

9. Qureshi AI, Suri MFK, Ringer AJ, Guterman LR, Hopkins LN (2002) Regional intraparenchymal pressure differences in experimental intracerebral hemorrhage: effect of hypertonic saline. Crit Care Med 30:435-441. https://doi.org/10.1097/00003246-20020 2000-00028

10. Rieger A, Rainov NG, Sanchin L, Ebel H, Furka I, Görömbey Z, Burkert W (1999) Is it useful to measure supratentorial ICP in the presence of a posterior fossa lesion? Absence of transtentorial pressure gradients in an animal model. Br J Neurosurg $13: 454-458$

11. Rosenwasser RH, Kleiner LI, Krzeminski JP, Buchheit WA (1989) Intracranial pressure monitoring in the posterior fossa: a preliminary report. J Neurosurg 71:503-505. https://doi.org/10. 3171/jns.1989.71.4.0503

12. Slavin KV, Misra M (2003) Infratentorial intracranial pressure monitoring in neurosurgical intensive care unit. Neurol Res 25:880-884. https://doi.org/10.1179/016164103771954014

13. Vanaclocha V, Sáiz-Sapena N, Rivera-Paz M, Herrera JM, OrtizCriado JM, Verdu-López F, Vanaclocha L (2017) Can we safely monitor posterior fossa intracranial pressure? A cadaveric study. Br J Neurosurg 31:557-563. https://doi.org/10.1080/02688697. 2017.1332336

14. Wolfla CE, Luerssen TG, Bowman RM (1997) Regional brain tissue pressure gradients created by expanding extradural temporal mass lesion. J Neurosurg 86:505-510. https://doi.org/10.3171/jns. 1997.86.3.0505

15. Higgins JPT, Thompson SG, Deeks JJ, Altman DG (2003) Measuring inconsistency in meta-analyses. BMJ 327:557-560. https:// doi.org/10.1136/bmj.327.7414.557

16. Mokri B (2001) The Monro-Kellie hypothesis: Applications in CSF volume depletion. Neurology 56:1746-1748. https://doi.org/ 10.1212/WNL.56.12.1746

17. Wilkinson HA (1990) Surgery and monitoring of traumatic intracerebral hematomas. Surg Neurol 33:296-297. https://doi.org/10. 1016/0090-3019(90)90054-s

18. Sahuquillo J, Poca MA, Arribas M, Garnacho A, Rubio E (1999) Interhemispheric supratentorial intracranial pressure gradients in head-injured patients: are they clinically important? J Neurosurg 90:16-26. https://doi.org/10.3171/jns.1999.90.1.0016

19. Weaver DD, Winn HR, Jane JA (1982) Differential intracranial pressure in patients with unilateral mass lesions. J Neurosurg 56:660-665. https://doi.org/10.3171/jns.1982.56.5.0660

20. Yano M, Ikeda Y, Kobayashi S, Otsuka T (1987) Intracranial pressure in head-injured patients with various intracranial lesions is identical throughout the supratentorial intracranial compartment. Neurosurgery 21:688-692. https://doi.org/10.1227/00006123198711000-00015

Publisher's note Springer Nature remains neutral with regard to jurisdictional claims in published maps and institutional affiliations. 